\title{
Resting-state frontostriatal functional connectivity in Parkinson's disease-related apathy
}

Baggio HC, MD, PhD ${ }^{a}$, Segura B, PhD ${ }^{a}$, Garrido-Millan JL, $\mathrm{MS}^{\mathrm{a}}$, Marti MJ, MD, PhD ${ }^{\mathrm{b}, \mathrm{c}, \mathrm{d}}$, Compta $\mathrm{Y}$, MD, PhD ${ }^{b, c, d}$, Valldeoriola F, MD, PhD ${ }^{b, c, d}$, Tolosa $E, M D^{b, c, d}$, Junque $C, P^{a} D^{a, b, d}$

a Departament de Psiquiatria i Psicobiologia Clínica, Universitat de Barcelona. Barcelona, Catalonia, Spain.

b Institut d'Investigacions Biomèdiques August Pi i Sunyer (IDIBAPS). Barcelona, Catalonia, Spain.

c Parkinson's Disease and Movement Disorders Unit, Neurology Service, Institut Clínic de Neurociències (ICN), Hospital Clínic de Barcelona. Barcelona, Catalonia, Spain.

d Centro de Investigación Biomédica en Red sobre Enfermedades Neurodegenerativas (CIBERNED), Hospital Clínic de Barcelona. Barcelona, Catalonia, Spain.

Word count:

Abstract: 240 Main text: 3352

Figures: 3 Tables: $2 \quad$ References: 43

Running title: Frontostriatal connectivity and apathy in PD

Keywords: Parkinson's disease, apathy, functional imaging, resting-state connectivity

\section{Disclosure:}

This work was funded by the Spanish Ministry of Science and Innovation [PSI2013-41393 grant to C.J., H.C.B. and B.S.], by the Generalitat de Catalunya [2014SG98], and an FI-DGR grant [2011FI_B 00045] to H.C.B., and CIBERNED.

The authors report no conflicts of interest. 


\section{Corresponding author:}

Prof. Carme Junqué

Department of Psychiatry and Clinical Psychobiology. University of Barcelona

Casanova 143 (08036) Barcelona, Spain

Phone: (+34) 934024570 // Fax: (+34) 934035294 // E-mail: cjunque@ub.edu

\section{Authors' contact information:}

HCB- $\underline{\text { hbaggio@ub.edu }}$ BS- $\underline{\text { bsegura@ub.edu JLGM-ilgarridomillan@gmail.com }}$

MJM- mjmarti@clinic.ub.es FV- fvallde@clinic.ub.es YC-ycompta@clinic.ub.es

ET- etolosa@clinic.ub.es $\quad C J$ - cjunque@ub.edu 


\section{ABSTRACT}

Background: One of the most common neuropsychiatric symptoms in PD is apathy, affecting between 23 and $70 \%$ of patients and thought to be related to frontostriatal dopamine deficits. In the present study, we assessed functional resting-state frontostriatal connectivity and structural changes associated with the presence of apathy in a large sample of PD subjects and healthy controls, while controlling for the presence of comorbid depression and cognitive decline.

Methods: Thirty-one healthy controls $(\mathrm{HC})$ and 62 age, sex and education-matched PD patients underwent resting-state functional MRI. Apathy symptoms were evaluated with the Apathy Scale (AS). The 11 Beck Depression Inventory-II items that measure dysphoric mood symptoms as well as relevant neuropsychological scores were used as nuisance factors in connectivity analyses. Voxel-wise analyses of functional connectivity between frontal lobes (limbic, executive, rostral motor and caudal motor regions), striata (limbic, executive, sensorimotor regions) and thalami were performed. Subcortical volumetry/shape analysis and frontosubcortical voxel-based morphometry were performed to assess structural changes.

Results: Twenty-five PD patients were classified as apathetic (PD-A) (AS>13). PD-A patients showed functional connectivity reductions compared with $\mathrm{HC}$ and with non-apathetic patients (PD-NA), mainly in left-sided circuits, and predominantly involving limbic striatal and frontal territories. Similarly, severity of apathy negatively correlated with connectivity in these circuits. No significant effects were found in structural analyses.

Conclusions: Our results indicate that the presence of apathy in PD is associated with functional connectivity reductions in frontostriatal circuits, predominating in the left hemisphere and mainly involving its limbic components. 


\section{INTRODUCTION}

Parkinson's disease (PD) has non-motor symptoms. Among them, one of the most common neuropsychiatric manifestations is apathy. Affecting between 23 and 70\% of PD patients,(1-3) apathy is characterized by behavioral (reduced goal-directed behavior), cognitive (lack of interest) and affective (flattened affect) symptoms.(2)

Although the pathophysiologic bases of apathy in PD are not clear, dopamine deficits affecting frontostriatal loops are thought to play an important role. Apathy is known to occur as a result of lesions affecting the medial and orbital parts of the prefrontal cortex and the portions of the basal ganglia connected to them, namely the ventral striatum.(4) In PD, severity of apathy has been found to correlate with frontal gray matter $(\mathrm{GM})(5)$ and ventral striatal volume reductions (6). Apathy following subthalamic nucleus stimulation surgery was shown to be associated with mesolimbic dopaminergic denervation,(7) and is amenable to dopaminergic therapy. $(8,9)$ These findings give support to the relevance of frontostriatal circuits in PDrelated apathy; to our knowledge, nonetheless, no published studies evaluated the presence of associated functional connectivity $(\mathrm{FC})$ changes.

The presence of apathy in PD is associated with cognitive deficits(10-12) and with a higher risk of dementia(13). Moreover, it often coexists with depression, and the symptomology of both syndromes overlaps(2) - specifically, symptoms of apathy occur as part of the depressive syndrome.(14) Although there's evidence to support that apathy exists as a distinct entity in $P D,(2,10,15-17)$ the study of its neural substrates, as well as its clinical detection, are complicated by such overlap.

Our aim in the present study was to evaluate resting-state FC and structural changes affecting the frontostriatal pathways in a large sample of PD patients and matched controls, while controlling for the associated effects of cognitive decline and depressive symptoms. We hypothesized that apathy in PD patients would be associated with disrupted FC in circuits, especially affecting the ventral striatum and the ventromedial prefrontal cortex. Considering that dopamine modulates frontostriatal FC, and that dopamine deficiency due to nigral degeneration precedes forebrain GM pathology in $\mathrm{PD},(18)$ we also expected that FC changes would be more marked than structural degeneration.

\section{METHODS}


Eighty-four non-demented PD patients and 38 healthy controls $(\mathrm{HC})$ matched for age, sex and years of education were included. Patients were recruited from the Parkinson's Disease and Movement Disorders Unit, Hospital Clínic de Barcelona. HC were recruited from individuals who volunteered to participate in scientific studies at the Institut de l'Envelliment, Universitat Autònoma de Barcelona. The inclusion criterion for patients was the fulfillment of the UK PD Society Brain Bank diagnostic criteria for PD (19). Exclusion criteria were: Mini-Mental State Examination scores $<25$ or dementia according to Movement Disorder Society criteria;(20) Hoehn and Yahr (HY) score >III; significant neurological, systemic or psychiatric (except depressive symptoms) comorbidity; pathological MRI findings other than mild white matter (WM) hyperintensities; root-mean-square head motion $>0.3 \mathrm{~mm}$ translation or $0.6^{\circ}$ rotation.

Four patients were excluded due to macroscopic movement, 14 due to head motion $>0.3 \mathrm{~mm}$ translation or $>0.6^{\circ}$ rotation, and one for being an outlier in connectivity analyses. Eight HC ( 2 due to microvascular WM changes, 5 due to incomplete filling of the AS) were excluded, leaving a final sample of $31 \mathrm{HC}$ and 65 PD patients. This subject sample was used in a recent FC study (21), and all except one HC and one PD patient were part of the sample used in a cortical thickness study (22).

All patients except one were taking antiparkinsonian drugs, consisting of different combinations of levodopa, cathecol-O-methyl transferase inhibitors, monoamine oxidase inhibitors, dopamine agonists and amantadine. All assessments were done while patients were in the on state. Levodopa equivalent daily dose (LEDD) was calculated as suggested by Tomlinson et al.(23) Motor disease severity was evaluated using HY and Unified Parkinson's Disease Rating Scale motor section (UPDRS) scores.

The study was approved by the institutional ethics committee, and all subjects provided written informed consent to participate.

\section{Neuropsychiatric evaluation}

Apathy symptoms were evaluated with the self-administered Apathy Scale (AS), recommended for use in PD.(24) Subjects were classified as apathetic if they scored $>13$.(15)

We also administered the Beck Depression Inventory-II (BDI) to all subjects. Kirsch-Darrow et al.(25) dissociated BDI items into 4 factors (apathy, dysphoric mood, loss of interest/pleasure, somatic factor); the loss of interest/pleasure factor was shown to be sensitive to symptoms of both depression and apathy, whereas the somatic factor can be influenced by other PD-related symptoms. The 11 items comprising the dysphoric mood factor loaded on negativity/sadness - 
symptoms not related to apathy -, and showed the lowest correlation with apathetic symptoms. We used the score in these 11 items (henceforth referred to as dysphoric mood score) as covariates of no interest in FC analyses to control for associated depression.

\section{Neuropsychological assessment}

Subjects underwent a thorough neuropsychological battery assessing cognitive functions frequently impaired in PD, using the following tests:

Attention/executive functions: backward minus forward digit spans; Trail-Making Test part A minus part B scores; phonemic fluency scores (words beginning with " $\mathrm{P}$ " produced in 60 seconds), and Stroop Color-Word Test interference scores). Visuospatial/visuoperceptual functions: Benton's Visual Form Discrimination and Judgment of Line Orientation tests. Memory: Rey's Auditory Verbal Learning Test total learning and 20-minute free recall scores. Composite z-scores for each cognitive function (referred to as $A / E$ scores, memory scores and VS/VP scores) were calculated as the mean of the z-scores of all tests within that function.

We have also investigated the presence of $\mathrm{MCl}$ following the Movement Disorder Society Task Force criteria, as described previously.(22)

\section{MRI acquisition}

Structural T1-weighted images, functional resting-state images and FLAIR images were acquired on a 3T Siemens MRI scanner as previously described (26).

\section{Processing of $f M R I$}

The preprocessing of resting-state images was performed with FSL (release 5.0.4, http://fsl.fmrib.ox.ac.uk/fsl/fslwiki/FSL) and AFNI (http://afni.nimh.nih.gov/afni). Briefly, it included removal of the first 5 volumes to allow for T1 saturation effects, skull stripping, grandmean scaling and temporal filtering $(0.01-0.1 \mathrm{~Hz})$. To control for the effect of subject head movement, physiological artifacts and other non-neural sources of signal variation on the estimation of connectivity, motion correction and regression of nuisance signals (six motion parameters, cerebrospinal fluid and WM) were performed. To remove the effects of images corrupted by motion, a scrubbing procedure as suggested by (27) was applied. Images were then smoothed with a 6-mm full-width at half maximum Gaussian kernel.

Additionally, head motion was calculated as the average Euclidean displacement between consecutive timepoints for rotatory and translatory motion (28). 


\section{Definition of regions of interest}

The frontal cortices were parcellated into limbic (anterior, posterior and medial orbital gyri, gyrus rectus, and subcallosal gyrus/ventral anterior cingulate), executive (rostral superior and middle frontal gyri and dorsal prefrontal cortex), rostral motor (caudal portions of lateral and medial superior frontal gyrus, caudal middle and inferior frontal gyrus) and caudal motor (precentral gyrus and caudal premotor area), as described by Tziortzi et al.(29) In this study, these frontal divisions were used as seeds for probabilistic tractography analyses that defined the functional striatal subregions included in the Oxford-GSK-Imanova Striatal Connectivity Atlas, which we used to parcellate the striata into limbic, executive and sensorimotor regions. We also included the thalami, defined using the Harvard-Oxford subcortical structural atlas. Supplementary Figure 1 displays the frontal and striatal segmentation scheme used.

To obtain each seed region's resting-state fMRI time series, the mask for each structure was non-linearly registered to each subject's T1-weighted image using FSL FNIRT, and subsequently linearly registered to native functional space.

\section{Functional connectivity analysis}

FC analyses were performed with FSL and AFNI. Initially, a mean time series was obtained from each seed region ( 4 frontal, 3 striatal, 1 thalamic per hemisphere) by averaging the time series of every voxel contained in it prior to smoothing, in native functional space. Subsequently, these time series were correlated with the time series of every voxel inside the regions of interest (ROI), thus producing a Pearson's $r$ coefficient correlation map. These were then converted to $\mathrm{z}$ maps using Fisher's r-to-z transformation.

\section{Cortical and subcortical gray matter volume analysis - voxel-based morphometry}

Structural data was analyzed with FSL-VBM,(30) a voxel-based morphometry (VBM)-style analysis. First, non-brain tissue from structural images was extracted. After segmentation, GM images were aligned to MNI152 standard space using affine registration. Resulting images were averaged to create a study-specific template, to which native GM images were then nonlinearly re-registered. The registered partial volume images were then modulated by dividing by the Jacobian of the warp field. The modulated segmented images were then smoothed with an isotropic Gaussian kernel (sigma=3 mm). A mask with the structures of interest (frontal cortices, striatal divisions and thalamus) was created to define a search volume for subsequent statistical testing. 


\section{Subcortical volumetry and shape analysis}

Subcortical structures of interest (accumbens, pallidum, caudate, putamen and thalamus) were segmented using FIRST as implemented in FSL. Since subcortical structural volumes scale with head size, we used FSL SIENAX to calculate intracranial volumes, to be entered as nuisance factors in volume analyses. For shape analysis, surface meshes were fitted for each region of interest as a vertex distribution model, modelling its shape. Multivariate testing was then performed on the 3-dimensional coordinates of each vertex, followed by multiplecomparison correction, thus allowing the detection of localized structural changes.(31)

\section{White-matter hyperintensity load analysis}

To detect and quantify global (periventricular + deep) WM hyperintensity load, we used an automated segmentation procedure(32). Results are given in normalized volumes taking brain volume into account.

\section{Statistical analysis}

Voxelwise general linear model was applied using non-parametric testing (5000 permutations) for connectivity and VBM, testing all voxels inside the regions of interest (frontal lobes, striata, thalami), as well as for shape analyses. To evaluate the association between the presence of apathy and changes in FC, GM volume or shape, we performed both intergroup comparisons and correlations (AS scores). Sex and dysphoric mood scores were entered as nuisance variables in all analyses. Since group differences were found for $A / E$ and memory scores, these variables were entered in intergroup analyses; for correlation analyses, memory scores were entered as a nuisance factor as they correlated with AS scores. Significance level was set at $p<.05$, corrected for multiple comparisons using false-discovery rate (FDR) control, applied to $p$-value maps obtained from threshold-free cluster enhancement (33).

Pearson's chi-square test was used to compare categorical variables (hand dominance, sex, HY stage). Student's t-test was used to compare clinical and connectivity data means between patient subgroups (PD-NA, PD-A). Three-level one-way ANOVAs were used to compare clinical and sociodemographic data between $\mathrm{HC}$ and patient subgroups. Three-level one-way ANCOVAs were used to compare subcortical volumes between HC and patient subgroups, controlling for variables that showed intergroup differences in the previous step. Pearson's correlation was used to evaluate the relationship between demographic, clinical and neuropsychological measures. Partial correlations were performed in the HC group and in the collapsed PD group to assess the relationship between subcortical volumes and AS scores, 
controlling for the variables that correlated significantly with them. Statistical significance threshold was set at $p<.05$.

\section{RESULTS}

\section{Neuropsychiatric assessment}

Twenty-six PD patients (41.3\%) were classified as apathetic (AS>13). Table 1 shows sociodemographic, clinical and head motion data, with intergroup comparisons. There were no intergroup differences in age or years of education, although there were significant sex differences (men tended to be overrepresented in the apathetic PD (PD-A) group). As expected, dysphoric mood scores were significantly higher in apathetic patients (PD-A) than in non-apathetic patients (PD-NA). LEDD and measures of disease severity (HY, UPDRS scores) or duration were not significantly different between PD-A and PD-NA, and did not correlate significantly with AS, BDI or dysphoric mood scores.

The proportion of PD patients taking antidepressant medication was similar in both groups ( 6 PD-NA, 5 PD-A, $p=0.702$, Pearson's chi-square $=0.146$ ); AS and neuropsychological scores were not significantly different between medicated and unmedicated patients.

AS scores significantly correlated with memory scores $(r=-0.36, p=.004)$, whereas no significant effects were found for $A / E(p=0.503)$ or MMSE $(p=0.694)$ scores. Dysphoric mood scores did not correlate significantly with cognitive scores.

Although PD-A patients had lower A/E scores than HC and lower memory scores than $\mathrm{HC}$ and PD-NA (Table 1), the proportion of patients with $\mathrm{MCl}$ was not significantly different between patient groups (14 in the PD-NA and 15 in the PD-A group; $p=0.120$, Pearson's chisquare=2.423).

\section{Fronto-striatal-thalamic functional connectivity analysis}

PD-A patients showed FC reductions ( $p<.05$, FDR-corrected) compared with HC and with PDNA, mainly in left sided circuits and involving limbic regions (see Table 2 and Figure 1). Compared with HC, PD-A displayed reduced FC between the limbic striatal division and the rest of the left striatum. Moreover, the FC between the limbic division of the left striatum and the left frontal lobe was significantly reduced in PD-A compared with PD-NA; in PD-A compared to 
$\mathrm{HC}$, there was suggestive evidence ( $p=.06$, FDR-corrected) of reduced connectivity in the left orbitofrontal cortex and inferior frontal gyrus.

Correlation analyses in the collapsed PD sample showed that AS scores correlated negatively with the FC between both limbic and executive divisions of the left striatum and the left frontal lobe, between the limbic region of the left frontal lobe and the left striatum, and between the caudal and rostral frontal lobe and right striatum. Additionally, AS scores correlated negatively with the FC between the different subdivisions of the left frontal lobe (see Figure 2 and Supplementary Table). These regions of significant correlation with AS scores displayed a marked overlap with the clusters of significant intergroup differences described above in the left frontal lobe and in the striata.

Significant intergroup/correlation analysis results are summarized schematically in Figure 3.

FC results were maintained after adding head movement parameters as nuisance factors.

No significant correlations were found in the HC group. To confirm that the connectivity differences observed were not due to the unequal sex distribution among groups, we performed additional connectivity analyses comparing male and female HC; no significant differences were observed. Additionally, we performed post-hoc correlation analyses separately in male and female PD patients (Supplementary Figure 2), revealing that the observed significant negative correlations between AS scores and frontostriatal functional connectivity were present in both groups.

\section{VBM, subcortical volume and shape analysis}

No significant group differences or correlations with AS scores were observed for GM volume or subcortical volume/shape.

\section{White-matter hyperintensity load}

There were no significant intergroup differences or correlations between connectivity values in the clusters of intergroup differences and WM hyperintensity load.

\section{DISCUSSION}

The main finding of our study is that the presence of apathy in PD is associated with restingstate FC reductions affecting frontostriatal circuits, predominantly in the left hemisphere. 
These changes were observed while controlling for the presence of associated depressive symptoms and cognitive impairment, and were not accompanied by significant structural changes, suggesting that frontostriatal FC disruption plays a relevant role in PD-related apathy.

Our results indicate that the occurrence of apathy in PD is accompanied by reduced restingstate FC mainly affecting the limbic divisions of the striatum and prefrontal cortex. These structures are central components of the brain's reward and motivation systems,(34) recently shown to be involved in PD-related apathy.(35) Moreover, the limbic division of the left striatum showed reduced connectivity with the ipsilateral frontal cortex and with the rest of the left striatum. The limbic striatum is hypothesized to influence motor activity as the limbicmotor interface;(36) Haber et al. found evidence that the mechanism through which this region influences other striatal regions - and, consequently, prefrontal and motor cortices involves a "striatonigrostriatal spiral" through connections with mesencephalic dopaminergic nuclei.(37) Dopamine deficits have been shown to result in reduced frontostriatal FC.(38) The loss of mesencephalic dopaminergic neurons might thereby lead to the intrastriatal, frontostriatal and, ultimately, frontofrontal connectivity disruptions found in our study. Finally, the worsening of apathetic symptoms during the off state(39) and after dopaminergic treatment reduction following deep-brain stimulation surgery,(8) as well as its improvement with dopaminergic treatment $(7,40)$ suggest that dopamine deficiency is involved in PD-related apathy.

Taken together with our results, the abovementioned findings provide evidence that reduced striato-frontal resting-state FC is associated with apathy in PD, and is probably mediated by dopamine deficits. Furthermore, the "striatonigrostriatal spiral" model links the connections between the limbic striatum and other striatal/frontal regions not only to ventral tegmental area (VTA) but also to substantia nigra pars compacta dopaminergic neurons.(37)

The FC correlates found in our study had a clear left-sided predominance. This finding, alongside recently published studies, indicates that laterality of neuropathological changes influences the risk of developing apathy. Cubo et al. (2012), assessing PD patients up to 2 years from diagnosis, found that subjects with left-predominant motor symptoms (i.e., with lesser left-sided dopamine deficits) were less likely to be apathetic.(3) In line with these observations, the striatal volume correlates for apathy described by Carriere et al. were more pronounced in left-hemisphere structures.(6) Furthermore, in a recent study, Porat et al. (2014) found that off-medication PD patients with greater left-sided dopamine deficits had impaired approach motivation - a symptom of apathy -, whereas right-sided predominance 
was associated with impaired loss avoidance (possibly related to impulse control disorders).(41) No such relationship was found in our sample, which may be related to the fact that most patients had bilateral disease at assessment.

GM atrophy, which was not found in our study, has been inconsistently reported in association with apathy in PD. Reijnders et al. described that the degree of apathy correlated with frontal, insular and parietal reductions in GM volume,(5) whereas Isella et al. failed to find structural correlates.(17) Carriere et al., on the other hand, studying dopamine-resistant apathy through shape analysis, found striatal volume reductions, mainly in the nucleus accumbens, but no cortical thinning.(6) These variable results may be a consequence of the distinct techniques used, different sample characteristics and different control over potential confounds such as cognitive deficits and motor disability.(42) Taking into consideration the known patterns of PD evolution, it is conceivable that apathy in early PD is mediated by disrupted frontostriatal FC secondary to mesencephalic dopaminergic neuron degeneration; and that, as the degenerative process progresses to forebrain structures, frontal and striatal atrophy acquire a more important pathophysiological role. Longitudinal and radionuclide imaging studies are necessary to confirm this hypothesis and help disentangle the neurochemical and anatomical substrates of apathy in PD. Studies assessing PD patients in the on and off states may also shed light on the interaction between dopaminergic medication and the underlying pathological changes on FC, and provide a better characterization of the pathophysiology of apathy in PD. Moreover, future structural connectivity studies may be useful in assessing the involvement of microstructural WM changes in the FC disruptions observed here.

Finally, apathy in our study was associated with worse cognitive performance, giving support to the association between apathy and cognitive deficits in PD.(10-12) Apathy was also more common in male patients, a finding that has been inconsistently reported. $(1,10,11)$

Some limitations must be considered when interpreting our results. Groups were not matched for depressive or cognitive status. Although we did correct all connectivity analyses for these variables, we cannot exclude that they influenced the results obtained. Nonetheless, considering the high coexistence of apathy and depression or cognitive decline, our study sample is probably representative of the general population of apathetic PD patients. Additionally, with the aim of using a neuroanatomically valid scheme for parcellating the frontostriatal circuits, we used data from a carefully-performed structural connectivity study.(29) The resulting ROls, however, are large, and their averaged functional time series may fail to reflect the activity of functionally distinct subregions, possibly reducing the 
sensitivity to localized connectivity disruptions. Finally, despite rigorous exclusion criteria, our study groups were not matched for head movement, which can affect FC parameters.(43) We have nonetheless applied several preprocessing steps to minimize these effects. The fact that results were maintained after adding movement parameters as covariates, and that the main group differences were found between HC and PD-A (which did not differ in head motion) suggests that the observed effects are not artifactual.

In conclusion, our findings suggest that apathy in PD is associated with reduced resting-state frontostriatal FC, mainly affecting left-hemispheric limbic/ventromedial regions but also extending to premotor and primary motor regions, even in the absence of significant structural degeneration and while controlling for associated depression and cognitive decline. These findings are compatible with the purported involvement of dopamine deficits in frontostriatal pathways in the genesis of apathy symptoms in PD. 


\section{ACKNOWLEDGMENT}

Without the support of the patients, their families and control subjects this work would have not been possible.

\section{AUTHOR ROLES:}

1. Research project: A. Conception, B. Organization, C. Execution;

2. Statistical Analysis: A. Design, B. Execution, C. Review and Critique;

3. Manuscript: A. Writing of the first draft, B. Review and Critique

Baggio: 1B, 1C, 2A, 2B, 3A; Segura: 1B, 1C, 2B, 2C, 3B; Garrido-Millán: 2B, 3B; Marti: 1B, 1C, 2C, 3B; Valldeoriola: 1B, 1C, 2C, 3B; Compta: 1B, 1C, 2C, 3B; Tolosa: 1B, 1C, 2C, 3B; Junque: 1A, 1B, 1C, 2A, 2B, 2C, 3B.

Financial Disclosures of all authors (for the preceding 12 months).

Baggio HC. Grants: Spanish Ministry of Education and Science PSI2010-16174; Catalonia Government, $22009 S G R 94$.

Segura B. Grants: Spanish Ministry of Education and Science PSI2010-16174; Catalonia Government, 22009SGR94. Employment: Postdoctoral position University of Barcelona.

Marti MJ. Grants: Fundació la Marató de TV3 2006 (N-2006-TV060510), Instituto de Salud Carlos III (FISS). PI041600. Employment: Hospital Clínic i Provincial de Barcelona.

Valldeoriola F. Grants: FIS (Instituto de Salud Carlos III), Advisory boards: Boehringer, Ingelheim, Solvay. Employment: Hospital Clínic i Provincial de Barcelona. 
Compta Y. Grants: FEP_Premios11 (Federación Española de Parkinson). PI041833. Employment: Hospital Clínic i Provincial de Barcelona, University of Barcelona

Tolosa E. Grants: European Project on Mendelian Forms of Parkinson's Disease (MEFOPA). CP-FP PI040438. Catalonia Government PI040510. Instituto de Salud Carlos III FISS CP06/00126. PI08015. Michael J. Fox Foundation for Parkinson Disease (MJFF) PI041639, PI042534, PI042549, PI042548. Spaniard Network for Research on Neurodegenerative Disorders: Center of Biomedic Investigation in Neurodegenerative Diseases (CIBERNED), PI2010/05. Employment: Hospital Clínic i Provincial de Barcelona, University of Barcelona

Junque C. Grants: Spanish Ministry of Education and Science PSI2010-16174 and PSI2013-41393 grants, Catalonia Government 22009SGR94 and 2014SGR98 grants, Intellectual Property Rights: Books Editorial: Masson, Sintesis, Glosa, Ariel, Ars Medica, Employment: Full Professor University of Barcelona. Garrido-Millán JL reports no disclosures. 


\section{REFERENCES}

1. Pedersen KF, Alves G, Brønnick K, Aarsland D, Tysnes O-B, Larsen JP. Apathy in drugnaïve patients with incident Parkinson's disease: the Norwegian ParkWest study. J Neurol. 2010 Feb;257(2):217-23.

2. Kirsch-Darrow L, Fernandez HH, Fernandez HF, Marsiske M, Okun MS, Bowers D. Dissociating apathy and depression in Parkinson disease. Neurology. $2006 \mathrm{Jul}$ 11;67(1):33-8.

3. Cubo E, Benito-León J, Coronell C, Armesto D. Clinical correlates of apathy in patients recently diagnosed with Parkinson's disease: the ANIMO study. Neuroepidemiology. 2012 Jan;38(1):48-55.

4. Levy R, Dubois B. Apathy and the functional anatomy of the prefrontal cortex-basal ganglia circuits. Cereb Cortex. 2006 Jul;16(7):916-28.

5. Reijnders JSAM, Scholtissen B, Weber WEJ, Aalten P, Verhey FRJ, Leentjens AFG. Neuroanatomical correlates of apathy in Parkinson's disease: A magnetic resonance imaging study using voxel-based morphometry. Mov Disord. 2010 Oct 30;25(14):231825.

6. Carriere N, Besson P, Dujardin K, Duhamel A, Defebvre L, Delmaire C, et al. Apathy in Parkinson's disease is associated with nucleus accumbens atrophy: A magnetic resonance imaging shape analysis. Mov Disord. 2014 May 10;00(00):1-7.

7. Thobois $\mathrm{S}$, Ardouin $\mathrm{C}$, Lhommée $\mathrm{E}$, Klinger $\mathrm{H}$, Lagrange $\mathrm{C}$, Xie J, et al. Non-motor dopamine withdrawal syndrome after surgery for Parkinson's disease: predictors and underlying mesolimbic denervation. Brain. 2010 Apr;133(Pt 4):1111-27.

8. Czernecki V, Schüpbach M, Yaici S, Lévy R, Bardinet E, Yelnik J, et al. Apathy following subthalamic stimulation in Parkinson disease: a dopamine responsive symptom. Mov Disord. 2008 May 15;23(7):964-9.

9. Thobois $\mathrm{S}$, Lhommée E, Klinger $\mathrm{H}$, Ardouin $\mathrm{C}$, Schmitt E, Bichon A, et al. Parkinsonian apathy responds to dopaminergic stimulation of D2/D3 receptors with piribedil. Brain. 2013 May;136(Pt 5):1568-77.

10. Dujardin K, Sockeel P, Devos D, Delliaux M, Krystkowiak P, Destée A, et al. Characteristics of apathy in Parkinson's disease. Mov Disord. 2007 Apr 30;22(6):778-84.

11. Pluck GC, Brown RG. Apathy in Parkinson's disease. J Neurol Neurosurg Psychiatry. 2002;73(6):636-43.

12. Butterfield LC, Cimino CR, Oelke LE, Hauser R a, Sanchez-Ramos J. The independent influence of apathy and depression on cognitive functioning in Parkinson's disease. Neuropsychology. 2010 Nov;24(6):721-30.

13. Dujardin K, Sockeel P, Delliaux M, Destée A, Defebvre L. Apathy may herald cognitive decline and dementia in Parkinson's disease. Mov Disord. 2009 Dec 15;24(16):2391-7. 
14. Marin RS. Differential diagnosis and classification of apathy. Am J Psychiatry. 1990 Jan;147(1):22-30.

15. Starkstein SE, Mayberg HS, Preziosi TJ, Andrezejewski P, Leiguarda R, Robinson RG. Reliability, validity, and clinical correlates of apathy in Parkinson's disease. J Neuropsychiatry Clin Neurosci. 1992 Jan;4(2):134-9.

16. Aarsland D, Larsen JP, Lim NG, Janvin C, Karlsen K, Tandberg E, et al. Range of neuropsychiatric disturbances in patients with Parkinson's disease. J Neurol Neurosurg Psychiatry. 1999 Oct;67(4):492-6.

17. Isella V, Melzi P, Grimaldi M, lurlaro S, Piolti R, Ferrarese $C$, et al. Clinical, neuropsychological, and morphometric correlates of apathy in Parkinson's disease. Mov Disord. 2002 Mar;17(2):366-71.

18. Braak H, Tredici K Del, Rüb U, de Vos R a. ., Jansen Steur EN., Braak E. Staging of brain pathology related to sporadic Parkinson's disease. Neurobiol Aging. 2003 Mar;24(2):197-211.

19. Daniel SE, Lees AJ. Parkinson's Disease Society Brain Bank, London: overview and research. J Neural Transm Suppl. 1993 Jan;39:165-72.

20. Emre M, Aarsland D, Brown R, Burn DJ, Duyckaerts C, Mizuno Y, et al. Clinical diagnostic criteria for dementia associated with Parkinson's disease. Mov Disord. 2007 Sep 15;22(12):1689-707; quiz 1837.

21. Baggio H-C, Segura B, Sala-Llonch R, Marti M-J, Valldeoriola F, Compta Y, et al. Cognitive impairment and resting-state network connectivity in Parkinson's disease. Hum Brain Mapp. 2014 Aug 28;

22. Segura B, Baggio HC, Marti MJ, Valldeoriola F, Compta Y, Garcia-Diaz Al, et al. Cortical thinning associated with mild cognitive impairment in Parkinson's disease. Mov Disord. 2014 Aug 7;

23. Tomlinson CL, Stowe R, Patel S, Rick C, Gray R, Clarke CE. Systematic review of levodopa dose equivalency reporting in Parkinson's disease. Mov Disord. Birmingham Clinical Trials Unit, University of Birmingham, Edgbaston, Birmingham, United Kingdom. c.l.tomlinson@adf.bham.ac.uk DOI - 10.1002/mds.23429 SRC - Pubmed ID2 - 21069833 FG - 0; 2010 Nov 15;25(15):2649-53.

24. Leentjens AFG, Dujardin K, Marsh L, Martinez-Martin P, Richard IH, Starkstein SE, et al. Apathy and anhedonia rating scales in Parkinson's disease: critique and recommendations. Mov Disord. 2008 Oct 30;23(14):2004-14.

25. Kirsch-Darrow L, Marsiske M, Okun MS, Bauer R, Bowers D. Apathy and depression: separate factors in Parkinson's disease. J Int Neuropsychol Soc. 2011 Nov;17(6):105866.

26. Baggio H, Sala-Llonch R, Segura B, Marti M-J, Valldeoriola F, Compta Y, et al. Functional brain networks and cognitive deficits in Parkinson's disease. Hum Brain Mapp. 2014 Mar 17; 
27. Power JD, Barnes KA, Snyder AZ, Schlaggar BL, Petersen SE. Spurious but systematic correlations in functional connectivity MRI networks arise from subject motion. Neuroimage. 2012 Feb 1;59(3):2142-54.

28. Liu $Y$, Liang $M$, Zhou $Y$, He $Y$, Hao $Y$, Song $M$, et al. Disrupted small-world networks in schizophrenia. Brain. 2008 Apr;131(Pt 4):945-61.

29. Tziortzi AC, Haber SN, Searle GE, Tsoumpas C, Long CJ, Shotbolt P, et al. Connectivitybased functional analysis of dopamine release in the striatum using diffusion-weighted MRI and positron emission tomography. Cereb Cortex. 2014 May;24(5):1165-77.

30. Douaud G, Smith S, Jenkinson M, Behrens T, Johansen-Berg H, Vickers J, et al. Anatomically related grey and white matter abnormalities in adolescent-onset schizophrenia. Brain. 2007 Sep;130(Pt 9):2375-86.

31. Patenaude B, Smith SM, Kennedy DN, Jenkinson M. A Bayesian model of shape and appearance for subcortical brain segmentation. Neuroimage. 2011 Jun 1;56(3):907-22.

32. Ithapu V, Singh V, Lindner C, Austin BP, Hinrichs C, Carlsson CM, et al. Extracting and summarizing white matter hyperintensities using supervised segmentation methods in Alzheimer's disease risk and aging studies. Hum Brain Mapp. 2014 Feb 7;4235(August 2013):4219-35.

33. Smith SM, Nichols TE. Threshold-free cluster enhancement: addressing problems of smoothing, threshold dependence and localisation in cluster inference. Neuroimage. 2009 Jan 1;44(1):83-98.

34. Haber SN. Neuroanatomy of Reward: A View from the Ventral Striatum. In: Gottfried J, editor. Neurobiology of Sensation and Reward. Boca Raton (FL): CRC Press; 2011.

35. Martínez-Horta S, Riba J, de Bobadilla RF, Pagonabarraga J, Pascual-Sedano B, Antonijoan RM, et al. Apathy in Parkinson's disease: neurophysiological evidence of impaired incentive processing. J Neurosci. 2014 Apr 23;34(17):5918-26.

36. Mogenson GJ, Jones DL, Yim CHIYIU. From motivation to action: functional interface between the limbic system and the motor system. Prog Neurobiol. 1980 Jan;14(23):69-97.

37. Haber SN, Fudge JL, McFarland NR. Striatonigrostriatal pathways in primates form an ascending spiral from the shell to the dorsolateral striatum. J Neurosci. $2000 \mathrm{Mar}$ 15;20(6):2369-82.

38. Nagano-Saito A, Leyton M, Monchi O, Goldberg YK, He Y, Dagher A. Dopamine depletion impairs frontostriatal functional connectivity during a set-shifting task. J Neurosci. 2008 Apr 2;28(14):3697-706.

39. Czernecki V, Pillon B, Houeto JL, Pochon JB, Levy R, Dubois B. Motivation, reward, and Parkinson's disease: influence of dopatherapy. Neuropsychologia. 2002 Jan;40(13):2257-67. 
40. Devos D, Moreau C, Maltête D, Lefaucheur R, Kreisler A, Eusebio A, et al. Rivastigmine in apathetic but dementia and depression-free patients with Parkinson's disease: a double-blind, placebo-controlled, randomised clinical trial. J Neurol Neurosurg Psychiatry. 2014 Jun;85(6):668-74.

41. Porat O, Hassin-Baer S, Cohen OS, Markus A, Tomer R. Asymmetric dopamine loss differentially affects effort to maximize gain or minimize loss. Cortex. Elsevier Ltd; 2014 Feb;51:82-91.

42. Kostić VS, Filippi M. Neuroanatomical correlates of depression and apathy in Parkinson's disease: magnetic resonance imaging studies. J Neurol Sci. 2011 Nov 15;310(1-2):61-3.

43. Van Dijk KR a, Sabuncu MR, Buckner RL. The influence of head motion on intrinsic functional connectivity MRI. Neuroimage. Elsevier Inc.; 2012 Jan 2;59(1):431-8. 
Figure 1. Significant intergroup connectivity differences.

Color clusters indicate areas of significant ( $p<.05$, false-discovery rate correction) intergroup differences in connectivity with the seed regions indicated at the top, controlling for sex, dysphoric mood scores, memory scores and attention/executive scores. Corrected $p$-values are indicated in the color bar. MNI coordinates of the slices shown are indicated. Right hemisphere is shown on the left in axial and coronal views. SM: sensorimotor; $H C$ : healthy controls; $P D-A$ : Parkinson's disease patients with apathy; PD-NA: Parkinson's disease patients without apathy. 
Figure 2. Significant correlations between connectivity and apathy scale scores in the Parkinson's disease patient group.

Color clusters indicate areas of significant ( $p<.05$, false-discovery rate correction) negative correlation between apathy scores and connectivity with the seed regions indicated in the adjacent scatterplots ( $A$ : striatal seeds; $B$ : frontal seeds), controlling for sex, dysphoric mood scores and memory scores. Corrected $p$-values are indicated in the color bar. Scatterplots show the relationship between mean $\mathrm{z}$ connectivity values in the main significant clusters and apathy scale (AS) scores. Right hemisphere is shown on the left in axial and coronal views. 
Figure 3. Schematic summary of significant findings.

Left: significant intergroup connectivity differences (intergroup contrasts indicated by line color). Right: significant negative correlations between apathy scale scores and connectivity in the Parkinson's disease patient group. Continuous lines: results from frontal seed analyses; dashed lines: results from striatal seed analyses. Right hemisphere is depicted on the left. $H C$ : healthy control group; PD-A: Parkinson's disease patients with apathy; PD-NA: Parkinson's disease patients without apathy; $F L$ : frontal lobe, limbic division; FE: frontal lobe, executive division; FrM: frontal lobe, rostral motor division; FcM: frontal lobe, caudal motor division; StL: striatum, limbic division; StE: striatum, executive division; StSM: striatum, sensorimotor division. 
Table 1. Sociodemographic, clinical, head motion and white-matter hyperintensity characteristics of participants with intergroup comparisons.

\begin{tabular}{|c|c|c|c|c|c|}
\hline & $\begin{array}{c}\mathrm{HC} \\
\mathrm{n}=31\end{array}$ & $\begin{array}{c}\text { PD-NA } \\
\mathrm{n}=37\end{array}$ & $\begin{array}{l}\text { PD-A } \\
n=25\end{array}$ & Test stats/p & $\begin{array}{l}\text { Significant post-hoc } \\
\text { Bonferroni test }(p)\end{array}$ \\
\hline Age & $\begin{array}{l}64.55 \\
(9.21)\end{array}$ & $63.43(8.45)$ & 65.60 (12.89) & $.352 / .704$ & \\
\hline Education (yrs.) & $\begin{array}{l}11.00 \\
(4.28) \\
\end{array}$ & $11.16(5.39)$ & $8.88(3.57)$ & $2.118 / .126$ & \\
\hline Sex (male/female) & $15 / 16$ & $17 / 20$ & $20 / 5$ & $8.088 / .018 \chi$ & \\
\hline MMSE & $\begin{array}{l}29.68 \\
(0.48)\end{array}$ & $29.14(1.06)$ & $28.96(1.17)$ & $4.621 / .012$ & $\begin{array}{l}\mathrm{PD}-\mathrm{A}<\mathrm{HC}(.017), \\
\mathrm{PD}-\mathrm{NA}<\mathrm{HC}(.061)\end{array}$ \\
\hline AS & $7.31(4.44)$ & $7.49(3.60)$ & $19.36(4.13)$ & $79.742 /<.001$ & \multirow{3}{*}{$\begin{array}{c}\text { PD-A>HC (<.001), } \\
\text { PD-A>PD-NA }(<.001)\end{array}$} \\
\hline BDI & $6.37(5.90)$ & $7.22(4.44)$ & $14.8(5.39)$ & $21.497 /<.001$ & \\
\hline $\begin{array}{l}\text { Dysphoric mood } \\
\text { score }\end{array}$ & $2.16(2.49)$ & $2.32(2.33)$ & $5.60(2.90)$ & $15.834 /<.001$ & \\
\hline $\begin{array}{l}\text { Hand dominance } \\
(\mathrm{r} / \mathrm{l})\end{array}$ & $30 / 1$ & $36 / 1$ & $25 / 0$ & $.773 / .679 \chi$ & \\
\hline Disease duration & - & $7.54(5.52)$ & $7.24(4.13)$ & $.245 / .807+$ & \\
\hline UPDRS & - & $15.37(8.48)$ & $15.56(7.94)$ & $.087 / .931+$ & \\
\hline $\mathrm{HY}(\mathrm{I} / \mathrm{II} / \mathrm{III})$ & - & $13 / 22 / 2$ & $8 / 13 / 4$ & $1.921 / .383 \chi$ & \\
\hline LEDD (mg) & - & 687.8 (459.9) & $845.2(471.3)$ & $1.309 / .196 \dagger$ & \\
\hline A/E scores & $.09(.61)$ & $-.24(0.89)$ & $-.51(1.11)$ & $3.257 / .043$ & PD-A<HC (.040) \\
\hline Memory scores & $.06(.90)$ & $-.17(1.42)$ & $-1.16(1.07)$ & $8.142 / .001$ & $\begin{array}{c}\text { PD-A<HC }(.001), \\
\text { PD-A<PD-NA }(.005)\end{array}$ \\
\hline VS/VP scores & $.00(.80)$ & $-.41(.82)$ & $-.48(1.12)$ & $2.503 / .098$ & \\
\hline $\begin{array}{l}\text { Rotatory head } \\
\text { motion }(\mathrm{mm})\end{array}$ & $.03(.01)$ & $.05(.04)$ & $.04(.02)$ & $4.952 / .009$ & $\mathrm{PD}-\mathrm{NA}>\mathrm{HC}(.028)$ \\
\hline $\begin{array}{l}\text { Translatory head } \\
\text { motion (degrees) }\end{array}$ & $.08(.05)$ & $.07(.04)$ & $.07(.04)$ & $.428 / .653$ & \\
\hline $\begin{array}{l}\text { Normalized WM } \\
\text { hyperintensity } \\
\text { volume }\end{array}$ & $\begin{array}{c}799.2 \\
(1017)\end{array}$ & $\begin{array}{c}792.9 \\
(1354.4)\end{array}$ & $\begin{array}{l}1000.9 \\
(992.7)\end{array}$ & $.287 / .751$ & \\
\hline
\end{tabular}

Results are presented in means (SD). Statistically significant results $(p<.05)$ are marked in bold. $A S:$ apathy scale; BDI: Beck depression inventory-II; Disease duration: duration of motor symptoms, in years; UPDRS: unified Parkinson's disease rating scale, motor section; HY: Hoehn and Yahr scale; $L E D D$ : levodopa equivalent daily dose; $A / E$ : attention/executive; VS/VP: visuospatial/visuoperceptual; WM: white matter. Head motion refers to average Euclidean displacement between consecutive scans. HC: healthy controls; PD-NA: Parkinson's disease patients without apathy; $P D-A$ : Parkinson's disease patients with apathy. Test stats: F-statistics, Pearson's chi-square $(\chi)$ or Student's $t\left({ }^{\dagger}\right)$. 
Table 2. Significant intergroup connectivity differences.

\begin{tabular}{|c|c|c|c|c|c|}
\hline \multirow[b]{2}{*}{ Seed } & \multirow[b]{2}{*}{ Contrast } & \multirow[b]{2}{*}{$\begin{array}{l}\text { Volume } \\
\left(\mathrm{mm}^{3}\right)\end{array}$} & \multicolumn{2}{|c|}{ maximum } & \multirow[b]{2}{*}{ Topography } \\
\hline & & & $\begin{array}{c}\text { MNI } \\
\text { coordinates } \\
(x, y, z)\end{array}$ & $p$ value & \\
\hline \multirow{4}{*}{$\begin{array}{l}\text { Left limbic } \\
\text { frontal } \\
\text { lobe }\end{array}$} & $\mathrm{HC}>\mathrm{PD}-\mathrm{A}$ & 4077 & $-15,6,-6$ & .032 & $\begin{array}{c}\text { Left limbic/executive/SM } \\
\text { striatum }\end{array}$ \\
\hline & \multirow{3}{*}{$\begin{array}{c}\text { PD- } \\
\text { NA>PD-A }\end{array}$} & 2079 & $-27,-6,3$ & .016 & $\begin{array}{l}\text { Left executive/SM } \\
\text { striatum }\end{array}$ \\
\hline & & 1998 & $-15,-18,21$ & .033 & $\begin{array}{l}\text { Left executive/limbic } \\
\text { striatum }\end{array}$ \\
\hline & & 297 & $-18,12,-12$ & .044 & Left limbic striatum \\
\hline \multirow{2}{*}{$\begin{array}{l}\text { Right } \\
\text { limbic } \\
\text { frontal } \\
\text { lobe }\end{array}$} & $\begin{array}{c}\mathrm{HC}>\mathrm{PD}- \\
\mathrm{NA}\end{array}$ & 837 & $27,-3,-6$ & .005 & \multirow{2}{*}{$\begin{array}{l}\text { Right executive/limbic } \\
\text { striatum }\end{array}$} \\
\hline & $\mathrm{HC}>\mathrm{PD}-\mathrm{A}$ & 1404 & $27,0,-9$ & .005 & \\
\hline \multirow{9}{*}{$\begin{array}{l}\text { Left limbic } \\
\text { striatum }\end{array}$} & $\mathrm{HC}>\mathrm{PD}-\mathrm{A}$ & 2808 & $-21,-6,-6$ & .018 & $\begin{array}{c}\text { Left posterior } \\
\text { limbic/executive/SM } \\
\text { striatum }\end{array}$ \\
\hline & \multirow{8}{*}{$\begin{array}{c}\text { PD- } \\
\text { NA>PD-A }\end{array}$} & 6426 & $-36,-24,51$ & .045 & Left precentral gyrus \\
\hline & & 3672 & $-27,27,21$ & .045 & Left frontal pole \\
\hline & & 1431 & $-18,54,9$ & .045 & $\begin{array}{l}\text { Left paracingulate, frontal } \\
\text { pole }\end{array}$ \\
\hline & & 1431 & $-36,27,-18$ & .045 & Left OFC \\
\hline & & 702 & $-60,-6,39$ & .045 & \multirow{4}{*}{ Left PCG } \\
\hline & & 648 & $-9,-18,78$ & .045 & \\
\hline & & 648 & $-60,3,15$ & .045 & \\
\hline & & 297 & $-39,54,12$ & .048 & \\
\hline \multicolumn{6}{|c|}{$\begin{array}{l}\text { Description of clusters (>10 voxels) of significant intergroup connectivity differences, } \\
\text { controlling for dysphoric mood, memory and attentional/executive scores, as well as sex. } H C \text { : } \\
\text { healthy controls; } P D-A \text { : Parkinson's disease patients with apathy; } P D-N A \text { : Parkinson's disease }\end{array}$} \\
\hline
\end{tabular}




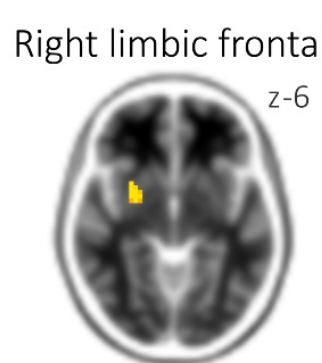

$\mathrm{HC}>\mathrm{PD}-\mathrm{NA}$

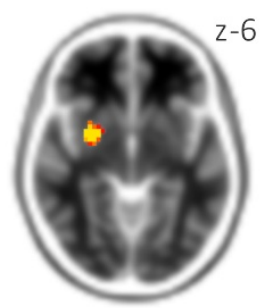

HC > PD-A
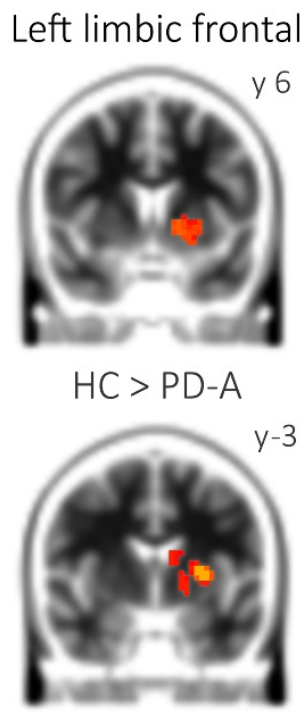

PD-NA > PD-A
Left limbic striatum

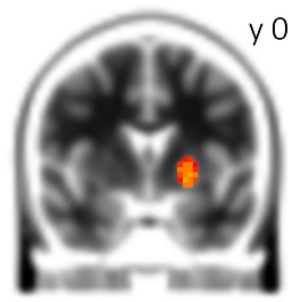

$\mathrm{HC}>\mathrm{PD}-\mathrm{A}$

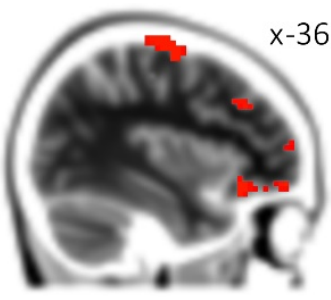

PD-NA > PD-A
Right SM striatum

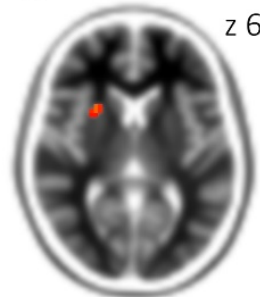

PD-NA > PD-A

p.05

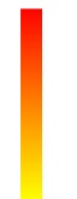

$p<.001$

Figure 1
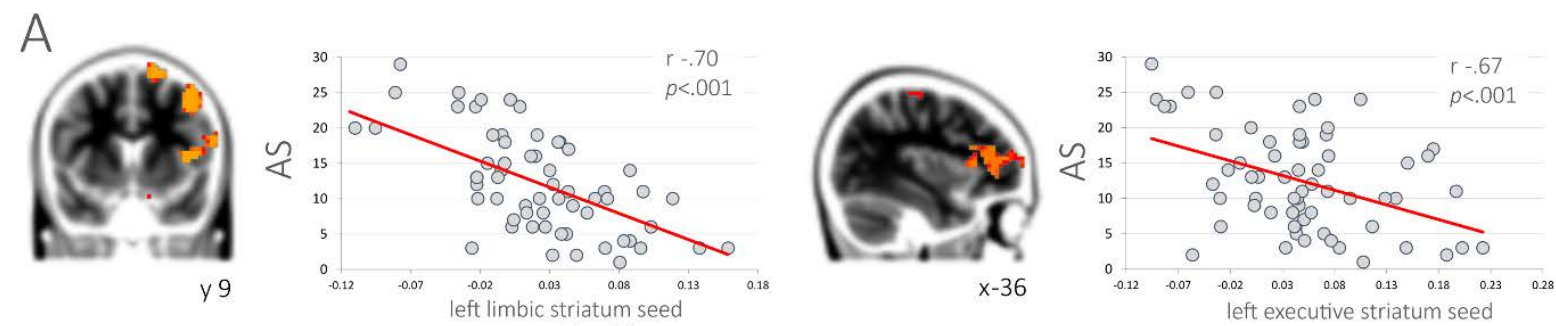
correlation (z)

left executive striatum seed correlation (z)
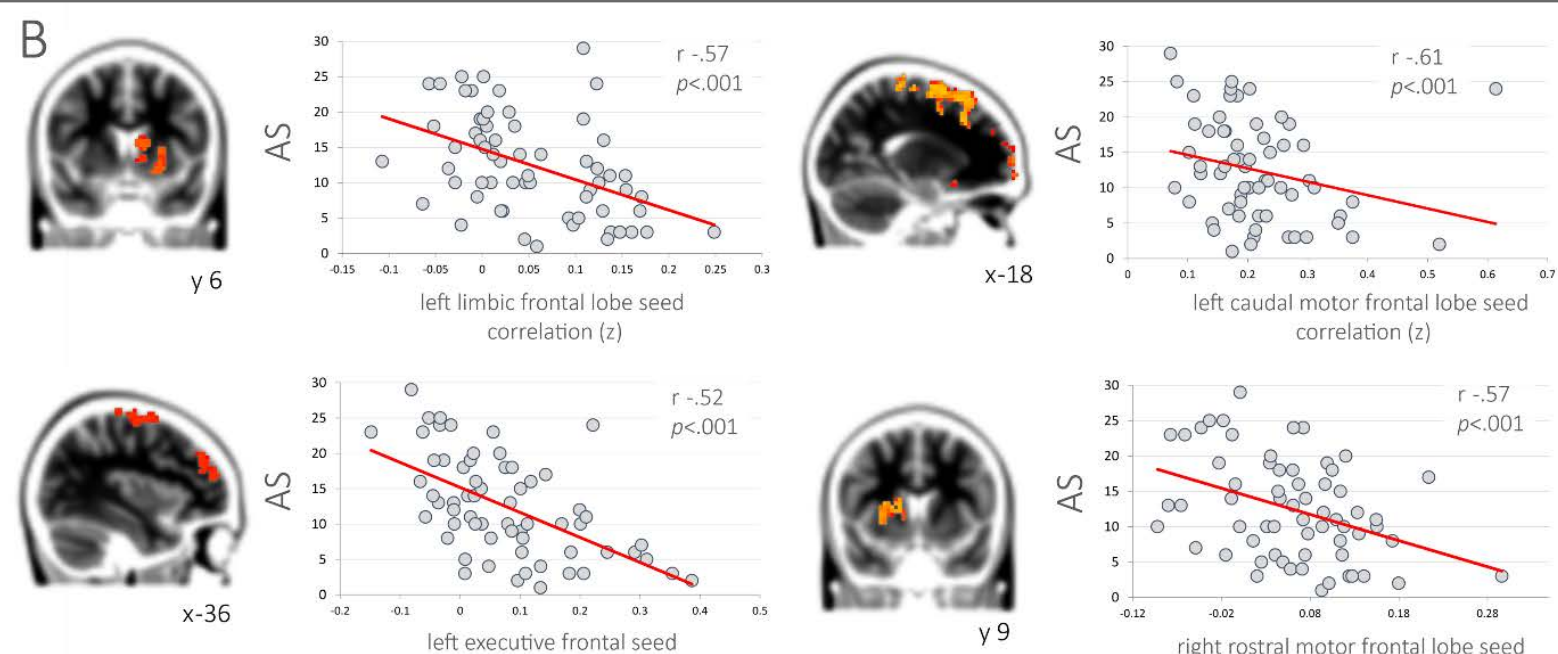
correlation (z)
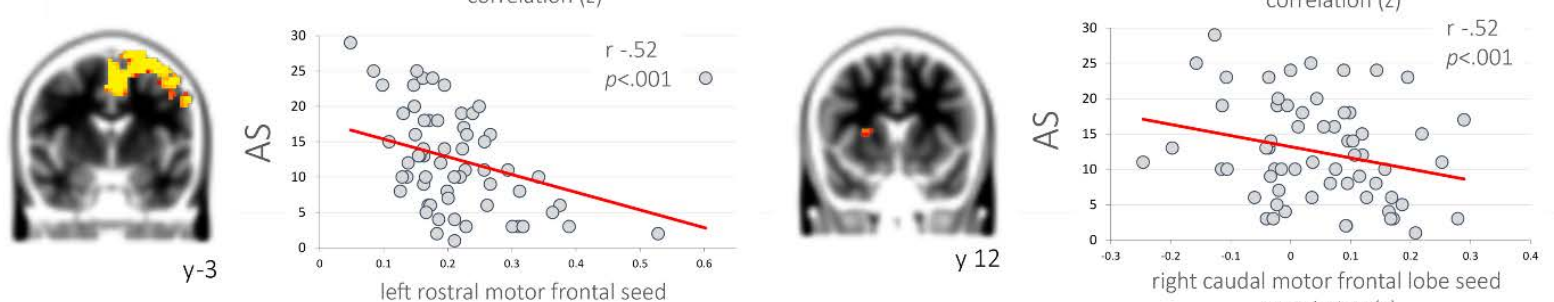
correlation ( $\mathrm{z}$ 


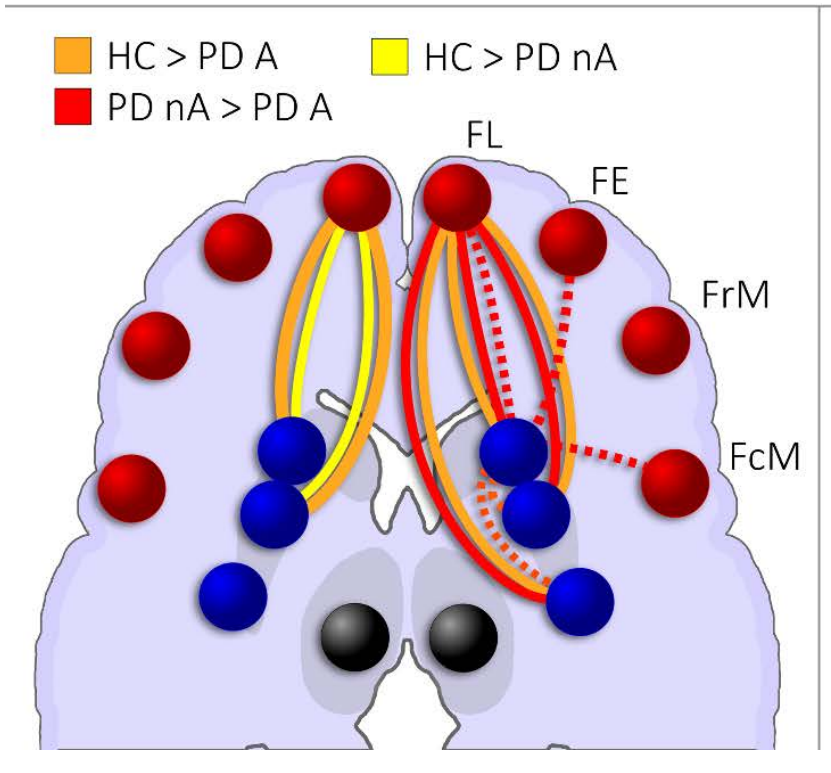

negative correlation AS

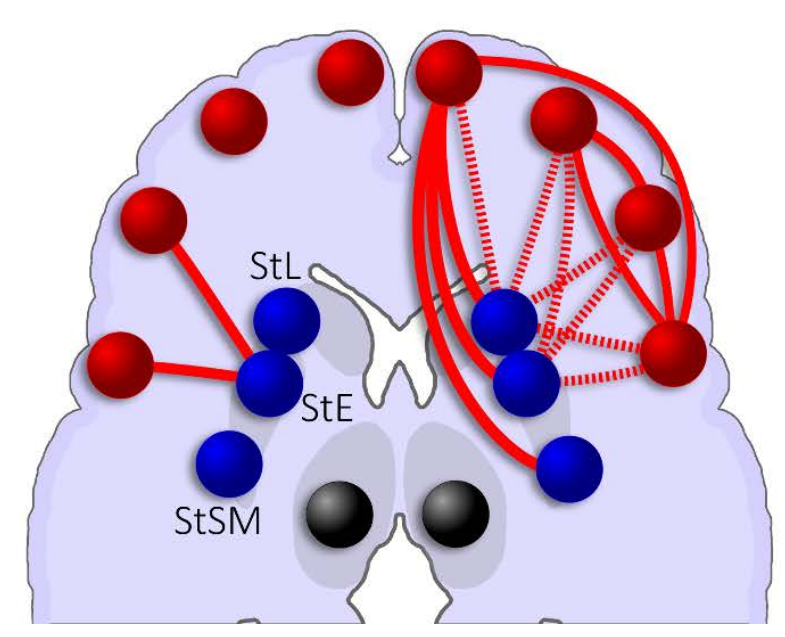

Figure 3 